\title{
Mucopolysaccharidoses: early diagnostic signs in infants and children
}

\author{
Cinzia Galimberti', Annalisa Madeo², Maja Di Rocco ${ }^{2}$ and Agata Fiumara ${ }^{3 *}$
}

\begin{abstract}
Mucopolysaccharidoses (MPS) comprise a group of lysosomal disorders that are characterized by progressive, systemic clinical manifestations and a coarse phenotype. The different types, having clinical, biochemical, and genetic heterogeneity, share key clinical features in varying combinations, including joint and skeletal dysplasia, coarse facial features, corneal clouding, inguinal or abdominal hernias, recurrent upper respiratory tract infections, heart valve disease, carpal tunnel syndrome, and variable neurological involvement. In the severe forms, these features usually appear in the first months of life, but a correct diagnosis is often reached later when suggestive signs are manifest. All MPS types may have severe or attenuated presentations depending on the residual enzymatic activity of the patient. Based on data from the literature and from personal experience, here we underline the very early signs of the severe forms which should alert the paediatrician on their first appearance. A few early signs are typical of MPS (i.e. gibbus) while many are unspecific (hernias, upper airway infections, organomegaly, etc.), and finding the association of many unspecific signs might prompt the paediatrician to search for a common cause and to carefully look for other more specific signs (gibbus and other skeletal deformities, heart murmur). We stress the need to increase awareness of MPS among paediatricians and other specialists to shorten the still existing diagnostic delay. A timely diagnosis is mandatory for the commencement of treatment as soon as possible, when available, to possibly obtain better results.
\end{abstract}

Keywords: Mucopolysaccharidosis, MPS, Early-onset MPS, Early MPS signs, Early MPS symptoms

\section{Background}

Mucopolysaccharidoses (MPS) are a group of clinically heterogeneous diseases caused by deficiencies of the lysosomal enzymes required for the breakdown of glycosaminoglycans (GAGs).

MPS are characterized by progressive and systemic clinical manifestations. Despite their biochemical and genetic heterogeneity, different types share key clinical features in varying combinations, including joint and skeletal dysplasia with stiffness (except MPS IV where there is laxity) and pain, coarse facial features, corneal clouding, inguinal or abdominal hernias, recurrent upper respiratory tract infections, heart valve disease, carpal tunnel syndrome, and variable neurological involvement. These features usually appear in the first months of life for the severe forms and

\footnotetext{
* Correspondence: agatafiumara@yahoo.it

Cinzia Galimberti and Annalisa Madeo contributed equally to this work.

Maja Di Rocco and Agata Fiumara are senior authors.

${ }^{3}$ Regional Referral Centre for Metabolic Diseases, Pediatric Clinic, Department

of Clinical and Experimental Medicine, University of Catania, AOU Policlinico

VE, Via Santa Sofia 78, 95123 Catania, Italy

Full list of author information is available at the end of the article
}

in early childhood for the more attenuated forms, but are often underestimated and generally taken into account only when clearly evident.

The rarity of these disorders and the variability in clinical presentation frequently leads to a diagnostic delay; this may range from months, for the severe forms, to years, for the attenuated ones (see Rigoldi et al. in this supplement [1]). Nevertheless, due to the fast progression and urgent need for intervention in the severe presentations, even months of delay in diagnosis may produce catastrophic results for the future health of the patient.

Our aim is to underline in this review the very early signs of the severe forms which should alert the paediatrician on their first appearance.

\section{What signs/symptoms can we expect in children with severe forms of MPS?}

Symptoms and signs suggestive of different forms of MPS are reported in Table 1 according to age. In the first 6 months of life, inguinal hernias, abnormally frequent respiratory infections, otitis, and organomegaly can be seen 
Table 1 Age of onset of the main signs and symptoms in different mucopolysaccharidosis (MPS) types

\begin{tabular}{|c|c|c|c|}
\hline Sign/ symptom & $\begin{array}{l}\text { Age of onset } \\
0-24 \text { months }\end{array}$ & $\begin{array}{l}\text { Age of onset } \\
>2 \text { yrs }\end{array}$ & MPS \\
\hline Hydrops foetalis $[10,19]$ & Birth & & I, IVA, VII \\
\hline Diarrhea $[6,13]$ & First months & & I, II, III \\
\hline Hernias $[11,12]$ & $\begin{array}{l}\text { Birth or first } \\
\text { months }\end{array}$ & & $\begin{array}{l}\text { Almost all } \\
\text { MPS }\end{array}$ \\
\hline $\begin{array}{l}\text { Upper respiratory } \\
\text { hypersecretion/ } \\
\text { Infections }[6,13]\end{array}$ & $\begin{array}{l}\text { First months } \\
-1 \text { Year }\end{array}$ & & $\mathrm{I}, \mathrm{II}, \mathrm{III}, \mathrm{Vl}$ \\
\hline \multirow{2}{*}{$\begin{array}{l}\text { Heart valve } \\
{[8,13,18,21]}\end{array}$} & $<1$ year & & I \\
\hline & & $<5$ yrs & $\| \mathrm{II}, \mathrm{VII}$ \\
\hline \multirow[t]{2}{*}{ Coarse face $[11,15]$} & $9-18 m$ & & $\mathrm{I}, \mathrm{Il}, \mathrm{Vl}$ \\
\hline & & $2-4$ yrs & $\|\|$, \\
\hline Corneal clouding [11] & $<15 \mathrm{~m}$ & & $\mathrm{I}, \mathrm{VI}$ \\
\hline $\begin{array}{l}\text { Liver/spleen } \\
\text { enlargement }[6,13]\end{array}$ & $<1$ year & & $\mathrm{I}, \mathrm{II}, \mathrm{II}, \mathrm{Vl}$ \\
\hline \multirow[t]{2}{*}{ Cognitive delay $[2,12]$} & $<18$ months & & I \\
\hline & & 2-3 years & $\|\|$, \\
\hline Macrocrania $[1,6,21]$ & Birth - 1 year & & $\mathrm{I}, \| \mathrm{I}, \mathrm{II}, \mathrm{IV}, \mathrm{VI}, \mathrm{VI}$ \\
\hline \multirow[t]{2}{*}{ Skeletal dysplasia $[2,15]$} & $<1$ year & & $\|, I V$ \\
\hline & & $2-4$ yrs & $\mathrm{I}, \mathrm{VI}$ \\
\hline \multirow[t]{2}{*}{ Growth impairment [13] } & $12-18 m$ & & I \\
\hline & & $<3$ yrs & $\mathrm{IV}, \mathrm{VI}$ \\
\hline \multirow[t]{2}{*}{ Behavioural anomalies $[1,6]$} & $<2$ yrs & & I \\
\hline & & $2-4$ years & $\|\|$, \\
\hline Sleep disturbances $[6,13]$ & First year & & $|| I,,|l|, V \mid$ \\
\hline
\end{tabular}

in MPS patients, particularly in MPS I (Hurler syndrome) which has the most precocious severe onset. Moreover, from 6 to 12 months, these infants very often develop a gibbus (toraco-lumbar kyphosis), heart murmur, umbilical hernia, mild hypotonia, and growth delay [2]. They may also have the typical coarse facial appearance (Fig. 1). MPS II, VI, and VII may present with a similar early phenotype. MPS IV patients have an early skeletal phenotype with the appearance of hip dysplasia in the first months of life and pigeon chest (pectus carinatum) in the first 12-18 months. MPS II infants present with a similar involvement but with later onset. In the second year of life, MPS I Hurler syndrome infants develop cognitive delay while an MPS II patient might be identified only because of overgrowth, frequent airway infections, and multiple surgeries $[3,4]$; the typical facial features appear only later. Some MPS II patients also develop hyperactivity between 18 and 24 months. In the third year of life, hyperactivity and cognitive delay are easily recognizable in MPS II and MPS III.

In summary, skeletal manifestations are typical and sometimes precocious. Coarse facies and cognitive delay are not early prominent signs.
What are the different presentations of the various types of MPS?

\section{MPS with somatic and cognitive involvement}

Mucopolysaccharidosis type I (MPS I; OMIM \#252800)

(Figs. 1, 2, 3, 4 and 5) is caused by a deficiency of the lysosomal hydrolase $\alpha$-L-iduronidase, which leads to multisystem accumulation of two GAGs: dermatan sulphate (DS) and heparan sulphate (HS) [3]. Based on its severity, MPS I is traditionally divided into three clinical phenotypes; however, these forms represent a continuum of disease severity. Hurler syndrome, the most severe form, typically presents during the first year of life. Affected children rapidly develop significant cognitive impairment and somatic disease in multiple organ systems, leading to death within the first decade in the absence of treatment. The attenuated forms of MPS I, known as Hurler-Scheie syndrome and Scheie syndrome, respectively, are characterized by later onset of symptoms, longer life expectancy, and mild or no central nervous system (CNS) involvement [2]. Inheritance is autosomal recessive, and in at least $50 \%$ of cases phenotype prediction is possible on the basis of

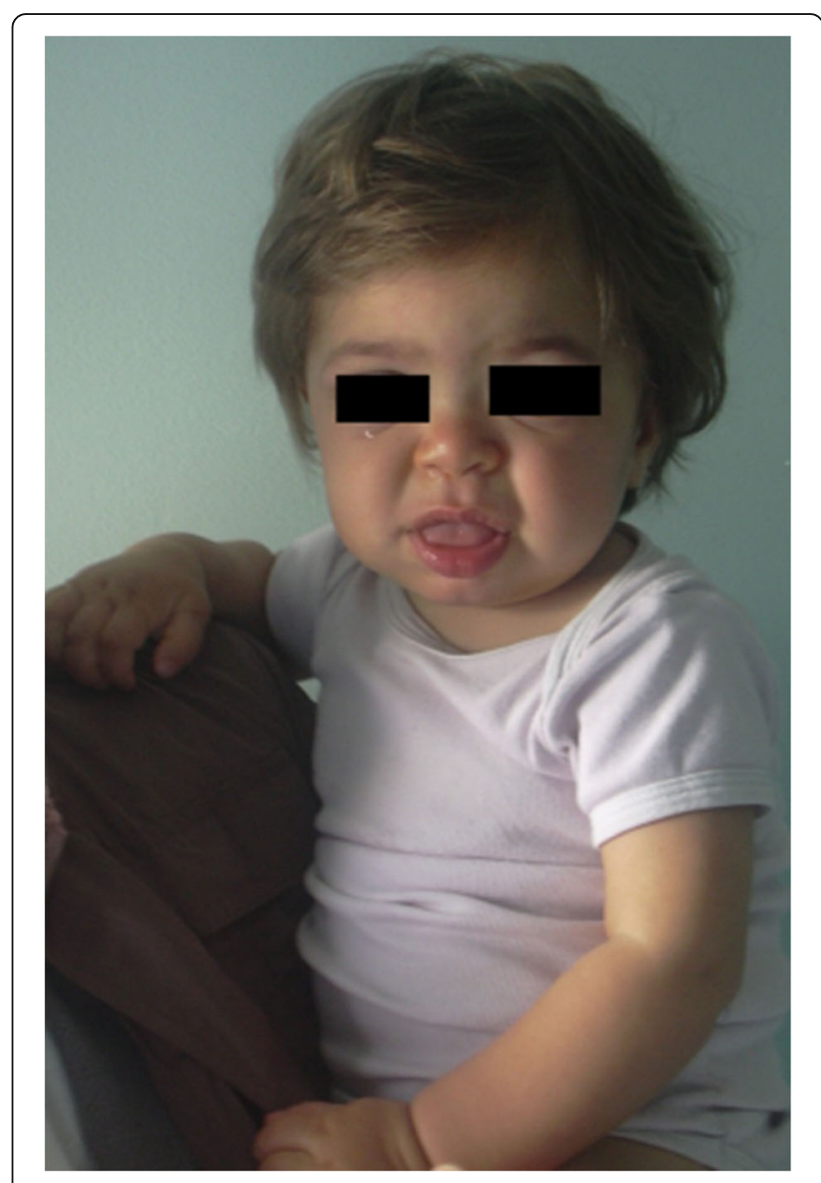

Fig. 1 MPS IH in 1-year-old patient. Coarse facies: flat nasal bridge, macroglossia, frontal bossing 


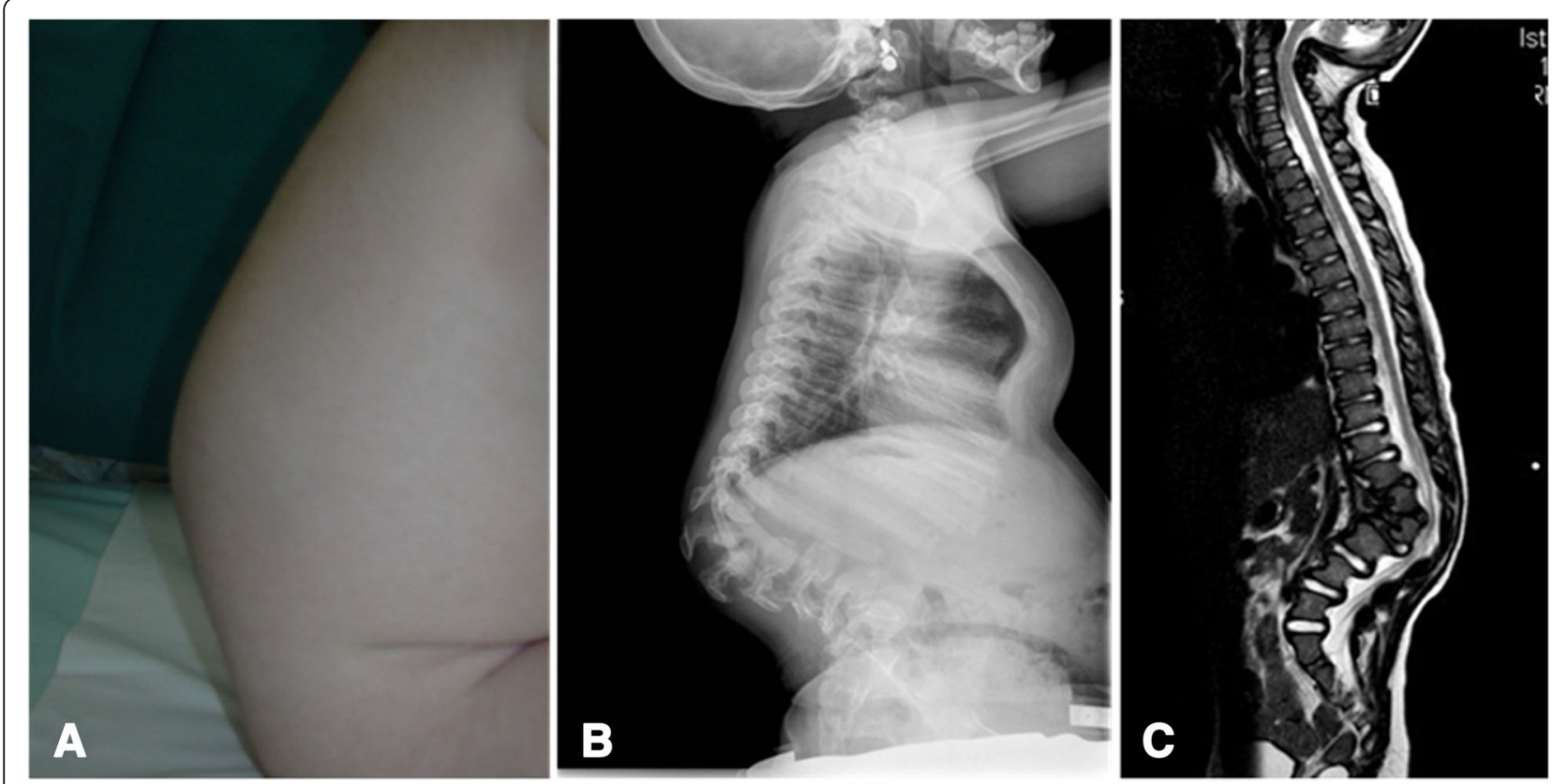

Fig. 2 MPS IH. a Gibbus in a 9-month-old patient. b Spine x-ray of the spine in a 3-year-old patient. c T2-weighted magnetic resonance image of the spine showing significant kyphosis and vertebral canal stenosis

genotype while in the remaining 50\% new mutations are detected [5]. Prevalence is approximately 1 in 100,000 live births [6].

Mucopolysaccharidosis type II (MPS II; OMIM \#309900) (Fig. 6), also known as Hunter syndrome, is the result of deficiency of the enzyme iduronate-2-sulfatase (I2S) with consequent GAG accumulation of DS and HS [7]. Prevalence is around 1 in 140,000-156,000 male live births [8]. Notably, this is the only X-linked MPS, and female carriers are usually asymptomatic; however, they can be exceptionally affected due to abnormalities of the X-chromosome, homozygosity, or skewed-X-inactivation [9]. The phenotypic expression also spans a wide spectrum of clinical severity. In severe cases, marked progressive neurologic involvement and somatic disease co-exist, and death usually occurs in the second decade of life. Other patients with minimal or no neurological dysfunction may present a severe somatic involvement while, at the opposite end of the spectrum, there are patients with only minimal somatic manifestations and normal intelligence who survive into adulthood [10].

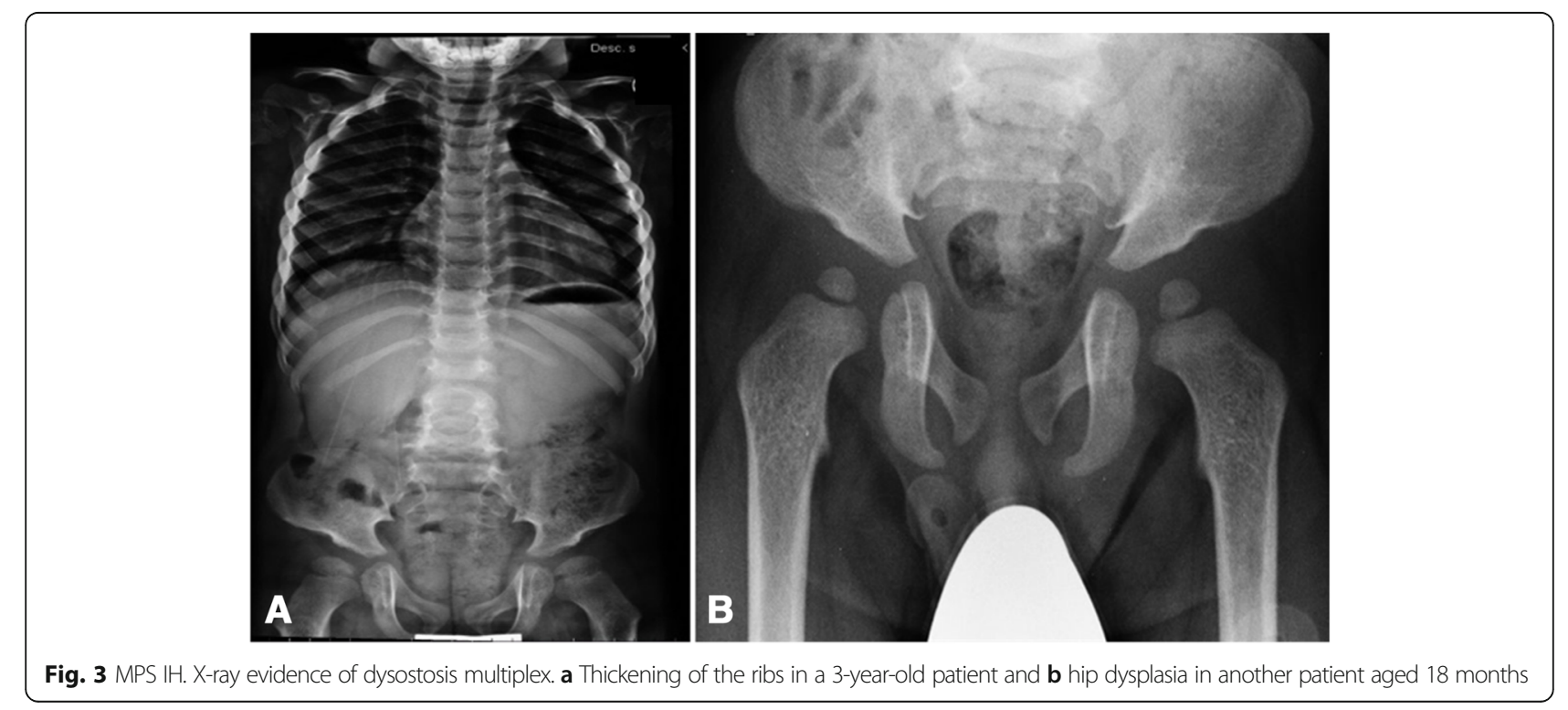




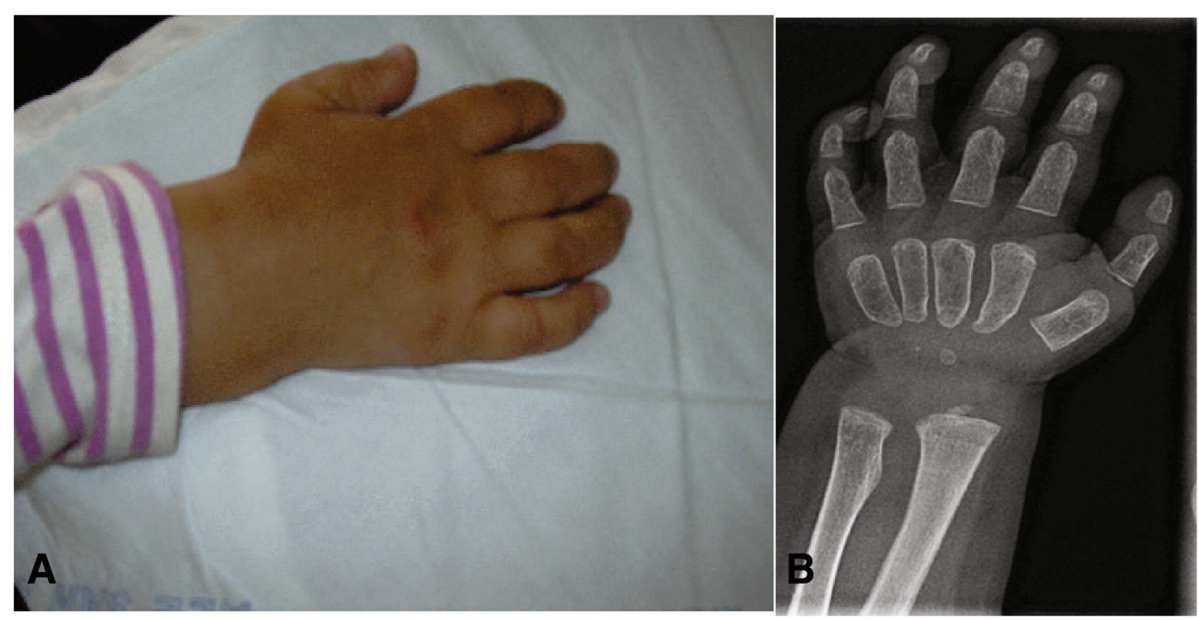

Fig. 4 MPS IH: a Claw hand in an 18 month-old patient; b hand X-ray.

Early signs and symptoms are shared by the severe forms of MPS I and II.

Sporadic cases of hydrops fetalis are described in MPS I [11], although, in general, these children appear normal at birth. The first signs of MPS I usually appear during the very first months of life, while those in MPS II usually occur a bit later [10]. Kiely et al. [2], in a retrospective study in 55 Hurler patients, observed that respiratory symptoms, feeding difficulties, inguinal hernia, and otitis media had appeared at a median age $\leq 6$ months, and kyphosis, cardiac abnormalities, enlarged head circumference, hypotonia, organomegaly, corneal clouding, joint restriction, and umbilical hernia appeared between 6 and 12 months. Umbilical hernia is one of the earliest presenting features in MPS I and II, and inguinal hernias are reported in approximately $60 \%$ of patients $[12,13]$.

Another common initial clue for MPS I and II can be upper airway recurrent infections with increased secretions.
Frequent otitis, chronic recurrent rhinitis, and persistent nasal discharge without obvious infection are not specific, but may help in strengthening the diagnostic suspicion if associated with more specific signs. Storage of GAG within the oro-pharynx leads to enlargement of the tonsils and adenoids, and contributes to upper airway complications. Another frequent finding is noisy breathing, particularly at night, associated in the later stages with obstructive sleep apnoea. As a consequence of frequent middle-ear infections and dysostosis of the ossicles of the middle ear, hearing loss is frequent, with both conductive and sensorineural deficits [8, 14]. Tracheobronchomalacia is commonly observed and can lead to acute airway obstruction or collapse, with this being one of the main causes of death in subsequent years [13].

Adenoidectomy, tonsillectomy, and tympanostomy, together with hernia repair, are the more frequent and early surgical interventions for MPS I and II patients,

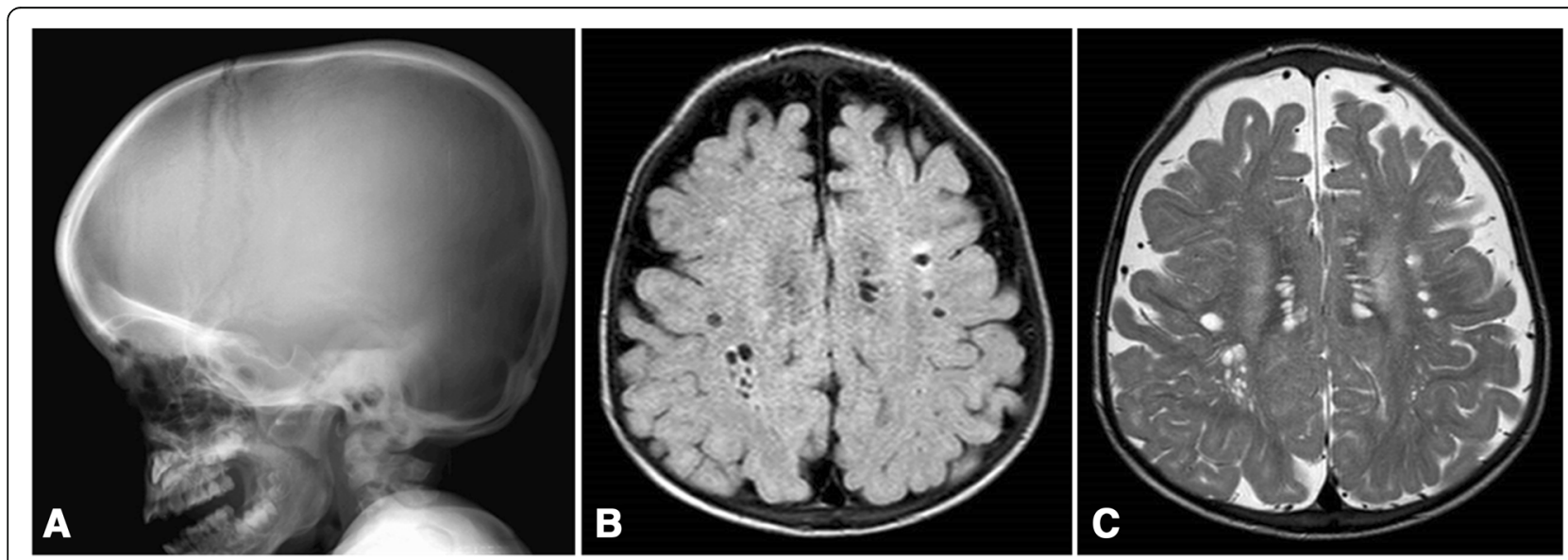

Fig. 5 MPS IH. a Thickening of the cortical bone of the skull and abnormal "J-shaped" conformation of the sella turcica. Dilatation of the periventricular spaces in $\mathbf{b}$ FLAIR and c T-2 weighted magnetic resonance images of a patient aged 17 months 


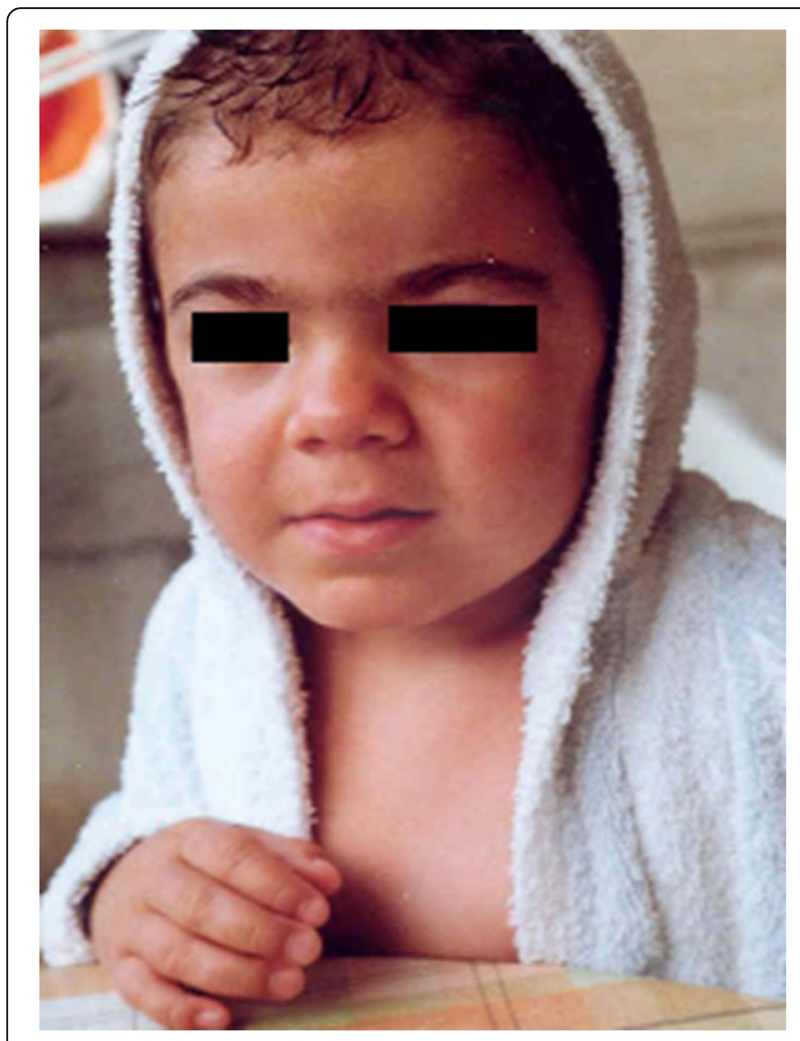

Fig. 6 MPS II. A 3-year-old patient with only mild characteristic facial features

often performed before knowing the diagnosis (in more than $50 \%$ of cases) [15].

Recurrent diarrhoea is quite common in early stages of MPS I, and can be also present in MPS II patients with neurologic involvement, while it is uncommon in the mildly affected patients $[2,9]$.

Gibbus deformity (dorso-lumbar kyphosis) (Fig. 2) becomes clinically apparent at a median age of 8.6 months to 1 year in MPS I [1, 12], representing a quite specific and precocious marker of MPS in general. Vertebral bodies appear flattened and beaked, potentially leading to later complications such as spinal nerve entrapment, acute spinal injury, and atlanto-occipital instability; thus, special care must be taken to prevent dislocation of the atlanto-axial joint during general anaesthesia and surgery. After 1 year of age, joint contractures and genu valgum represent other frequent signs of joint impairment and progressive skeletal dysplasia, which are easily detectable at earlier than 2 years of age in all infants affected by MPS. All these skeletal manifestations together, typical of MPS, are named dysostosis multiplex. Thickening of the ribs can be seen on radiographs (Fig. 3a), the long bones are short with wide shafts, and the knees are prone to valgus and varus deformities. Phalangeal dysostosis and synovial thickening lead to a characteristic claw hand deformity (Fig. 4), which is another typical feature that may raise suspicion of the disease. Hip dysplasia is frequently the reason why these children may be first seen by orthopaedic specialists. Typically, the pelvis is poorly formed, the femoral heads are small, and coxa valga is common, with progressive and debilitating hip deformity (Fig. 3b) [14, 16].

There is a notable difference between MPS I and MPS II; in MPS I, skeletal growth decelerates by 3 years of age [14], while MPS II patients show overgrowth in the first 5-6 years of age, slowing their growth thereafter [4].

Coarsening of the facial features (broad nose with flared nostrils, prominent supraorbital ridges, large rounded cheeks, thick lips, enlarged protruding tongue) caused by storage of GAGs in the soft tissues of the orofacial region and facial bones becomes apparent within the first 2 years, at a median age of 1.1 years for Hurler disease [12] and between 18 months and 4 years in the severe form of Hunter disease [16], although subtle phenotypic changes can be detected earlier (as early as the second half of the first year) by paediatricians with specific experience (Fig. 1). Macrocephaly becomes progressively more evident, and scaphocefaly is common (Fig. 5), but microcephaly is also possible in Hurler patients [17]. Facial and body hirsutism are always evident by the age of 24 months [14]. The skin may be thickened and inelastic. Notably, some MPS II patients develop a distinctive skin lesion, which is described as ivory-white papules (pebble-like) on the upper back and sides of the upper arms, pathognomonic of Hunter syndrome [18].

Cardiomyopathy and progressive thickening and stiffening of the valve leaflets in MPS I patients can lead to mitral and, less frequently, aortic regurgitation [14]. A murmur may be detected in the first months of life and has sometimes be reported as the first sign of the disease [19]. Cardiac involvement is also present in almost all patients with MPS II, but this starts at around 5 years of age [10]. Valve disease may lead to right and left ventricular hypertrophy and heart failure.

Protuberance of the abdomen caused by progressive hepatosplenomegaly is easily evident after 6 months of age, although storage of GAGs in the liver and spleen does not lead to organ dysfunction [14].

Corneal clouding occurs in almost all individuals with MPS I at earlier than 15 months of age [12], potentially causing severe visual impairment. On the contrary, corneal clouding is not a typical feature of Hunter syndrome [16], but slit-lamp examination may reveal discrete corneal lesions that do not affect vision. Retinal dysfunction may be detected, while glaucoma is not a common finding.

Early psychomotor development is generally detected as normal, but in MPS I developmental delay is usually obvious by the age of 18 months, with a progressive mental decline leading to a severe intellectual disability. Language skills are very limited in these children. A global delay of 
developmental milestones in MPS II becomes evident from 2 years onward [13]. However, the leading neurological feature of severe Hunter disease is represented by marked behavioural disturbances, such as hyperactivity, obstinacy, and aggressiveness, that are typically not observed in those with the attenuated phenotype [8].

Mucopolysaccharidosis type VII (MPS VII; OMIM \#253220), also known as Sly syndrome, is an ultra-rare MPS characterized by deficient activity of $\beta$-glucuronidase, with lysosomal storage of chondroitin sulphate (CS), DS, and HS, leading to cellular and organ dysfunction. The first patient was described by Sly et al. in 1973 [19] and a total of 145 patients have been reported in the literature up to now.

The clinical presentation and disease progression of MPS VII spans a wide severity spectrum, from early, severe, multisystem manifestations and death in the very first months, to a milder phenotype with later onset, normal or near-normal intelligence, and longer survival [20].

Phenotype characteristics of patients with MPS VII resemble those of MPS I and MPS II (short stature, skeletal dysplasia, macrocephaly, gingival hypertrophy, recurrent ear infections, hepatosplenomegaly, hernias, cardiac involvement, decreased pulmonary function, and cognitive impairment). An unexpectedly high proportion of described patients (41\%) have a history of non-immune neonatal hydrops fetalis (NIHF) [21]. Despite the prenatal onset of the disease in these patients, 13 out of 23 survived infancy with a mild to intermediate course into their late teens. Thus, the presence of NIHF does not, by itself, predict the possible severity of the clinical course if the patient survives infancy. Another early sign is macrocrania, while heart valve involvement and repeated infections of the upper and lower respiratory tract appear in the first 2 years of life. Moderate mental retardation and progressive hearing loss with speech impairment are also evident with time.

In summary, the severe forms of MPS I have a very early multisystem onset (within 6 months of life); MPS II is similar but with a later onset, and MPS VII has a prenatal onset with frequent NIHF and early severe involvement of the upper and lower respiratory tract.

\section{MPS with mostly neurological and cognitive involvement}

Mucopolysaccharidoses type III (MPS III, also known as Sanfilippo syndrome; Fig. 7) has a prevalent neurological presentation. MPS III is a lysosomal storage disorder caused by deficiency of one of the four enzymes involved in the catabolism of HS. Four different subtypes are known-MPS III type A (OMIM \#252900), type B (OMIM \#252920), type C (OMIM \#252930), and type D (OMIM \#252940)—each due to a different enzyme deficiency: type A, sulfamidase/ SGSH gene; type B, alfa- $N$-acetylglucosaminidase/NAGLU gene; type $\mathrm{C}$, alfa-glucosaminide $\mathrm{N}$-acetyltransferase/ HGSNAT gene; type D, $N$-acetilglucosamina-6-solfato sulfatasi/GNS gene). All four types (A to D) have autosomal recessive inheritance. MPS III is the most frequent of the MPS with an estimated prevalence between 0.3 and 4.1 cases for every 100,000 newborns, depending on the subtype and race [6].

Patients with Sanfilippo syndrome show progressive cognitive impairment in the second and third years of life. In contrast to the majority of MPS, somatic features are relatively less evident, and the CNS involvement is conspicuous. Speech development delay is usually the first sign noticed by the parents [22] but the main concerns can be behavioural problems (hyperactivity, anxious and aggressive behaviour, sleep disturbances), followed by progressive mental decline [22]. These symptoms can cause misdiagnosis as behavioural disturbances, attention deficit hyperactivity disorder (ADHD), and autism spectrum disorders [23]. Epilepsy may also be present.

Sleep disorders, the incidence of which is reported up to $80-90 \%$ [24], are a common feature. They consist of difficulties in falling asleep and frequent nocturnal awakening, with complete reversal of the day-night rhythm in some patients.

All these manifestations are very difficult to manage and have an enormous psychological impact on the quality of life for the whole family.

In general, although the phenotype can be very similar in the four subtypes, the clinical course in Sanfilippo B and $\mathrm{C}$ seems to be characterized by a less severe phenotype $[25,26]$.

Non-neurological symptoms are usually less pronounced in MPS III than in the other MPS. Recurrent ear, nose, and throat infections are observed at a younger age. Recurrent otitis, middle ear ossicle defects, and abnormalities in the inner ear can cause deafness. An excess of thick secretions and anatomical changes can produce obstruction of the airways. In the first years of life, diarrhoea is frequently reported, whereas constipation is more common in older patients.

Physical examination can show coarse facial features, although these are less pronounced. Patients have macrocephaly, broad eyebrows with medial flaring and synophrys, the hair is usually dry and coarse, and hypertrichosis is common. In younger patients, mild hepatomegaly and umbilical and inguinal hernias are frequently observed. Rare, and often mild, contractures are mostly found in the elbows. Growth is affected in approximately half of patients older than 12 years [25].

In summary, MPS III or Sanfilippo disease has a prominent neurological involvement with very mild somatic signs. Toddlers aged $2-3$ years old developing hyperactivity, ADHD, and aggressive behaviour may be suspected of having MPS III. 


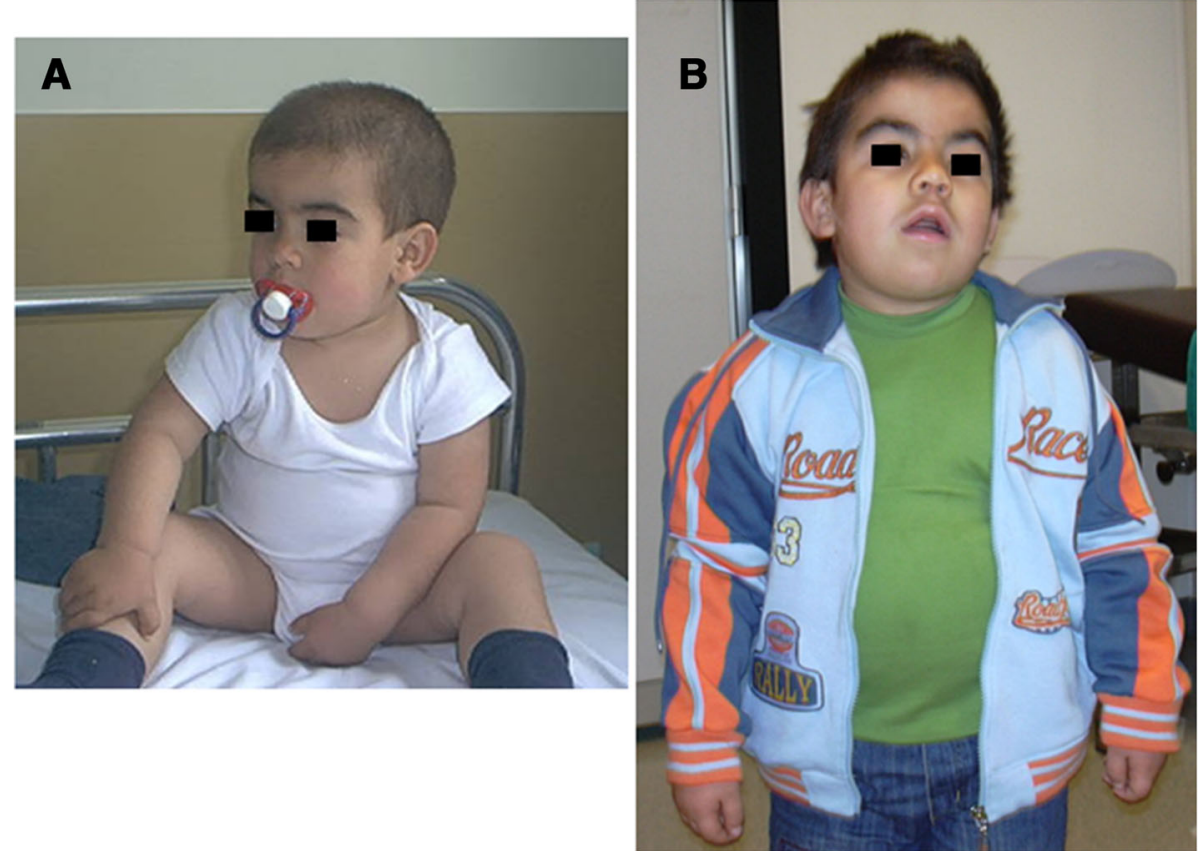

Fig. 7 MPS IIIA. The same patient at a 2.5 and $\mathbf{b} 5$ years old. Note the different face expression, showing progression of the neurological impairment

\section{MPS with prevalent somatic involvement}

MPS IV and MPS VI present with only somatic involvement. These are progressive conditions that spare intellectual ability and mainly affect the skeleton (MPS IVA), or all the organs/systems of the body (MPS VI). Inheritance is autosomal recessive. The rate at which symptoms worsen varies among affected individuals.

Mucopolysaccharidosis IV (MPS IV, also known as Morquio syndrome; Fig. 8) presents as two genetically distinct disorders, each with a different enzyme deficiency: MPS IVA (Morquio A syndrome; OMIM \#253000) due to deficiency of $\mathrm{N}$-acetylgalactosamine-6-sulfatase (GALNS gene), resulting in accumulation of keratan-sulphate (KS) and CS [26, 27]; and MPS IVB (Morquio B syndrome; OMIM \#253010) due to deficiency of beta-galactosidase activity leading to urine $\mathrm{KS}$ and oligosaccharide excretion as in GM1 patients. MPS IVB is indeed allelic to the various forms of GM1-gangliosidosis, but lacks the psychomotor deterioration seen in GM1 gangliosidosis [28, 29].

Patients with severe MPS IVA with no detectable enzyme activity reveal their first symptoms usually during the first year of life, although they are rarely diagnosed before 4-5 years of age. Information on the natural history of Morquio A patients is mainly derived from two wide-ranging collections of data [28]. Reduced growth rate begins at approximately 18 months of age and growth will stop at approximately 7 or 8 years of age. In contrast to the other MPS, Morquio A joints are usually lax and very flexible (hypermobile), but some joints (usually the hips and shoulders) may have a restricted range of motion. Patients with such skeletal involvement may receive a diagnosis of, or undergo evaluation for, spondyloepiphyseal dysplasia, pseudoachondroplasia, multiple epiphyseal dysplasia, or bilateral Legg-Calvé-Perthes disease [30].

A constant feature is odontoid hypoplasia; thus, dislocation of the atlanto-occipital joint can occur causing compression and damage to the bulbar and spinal cord, resulting in paralysis or even death if cervical stabilisation is not performed early.

MPS IVB patients have a very similar clinical manifestation but with a less prominent short stature. They have mildly coarse facial features, hearing loss, corneal opacities, valvular heart disease, and frequent upper respiratory tract infections. They may also present with inguinal hernia and mild hepatomegaly. Skeletal dysplastic features include platyspondyly, odontoid hypoplasia with possible cervical subluxation, kypho-scoliosis, coxa valga, and constricted iliac wings [28].

Mucopolysaccharidosis VI (MPS VI, also known as Maroteaux-Lamy syndrome; OMIM \#253200; Fig. 9) is due to pathogenic mutations in the arylsulfatase $\mathrm{B}(A R S B)$ gene located on chromosome 5. As a consequence, reduced or absent activity of the enzyme arylsulfatase B (ASB) ( $N$-acetylgalactosamine 4-sulfatase) impairs degradation of DS determining its cellular accumulation [31].

Patients with no detectable enzyme activity have the severe form of MPS VI and exhibit symptoms in early childhood. Their somatic phenotype is similar to Hurler syndrome. In most cases, by 2 or 3 years of age, severe 


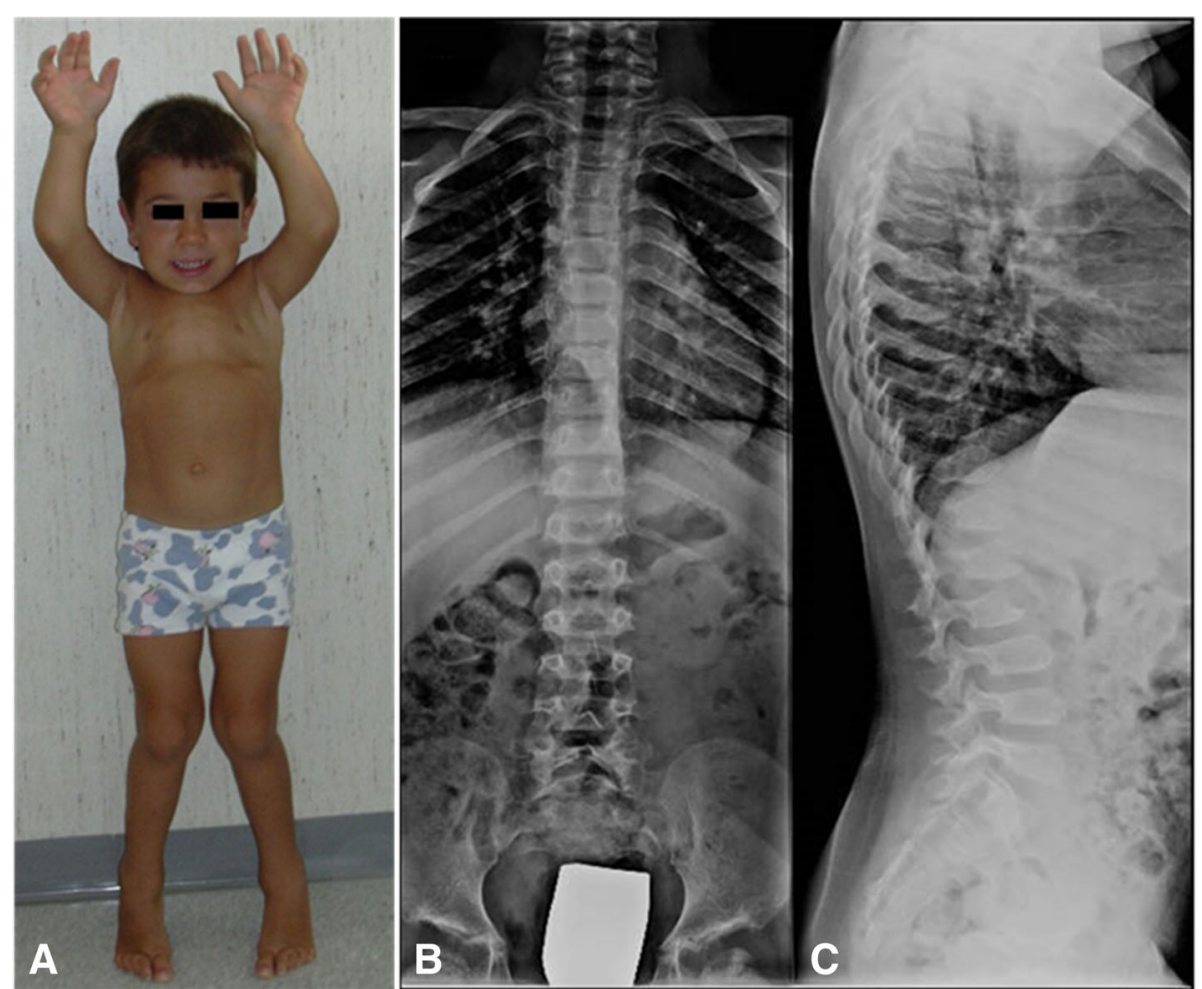

Fig. 8 MPS IVA. a 4 year-old patient with $\mathbf{b}$ antero-posterior and $\mathbf{c}$ lateral $x$-rays showing "paddle-shaped" ribs and flattened and rounded vertebrae with a typical "anterior beaking" aspect

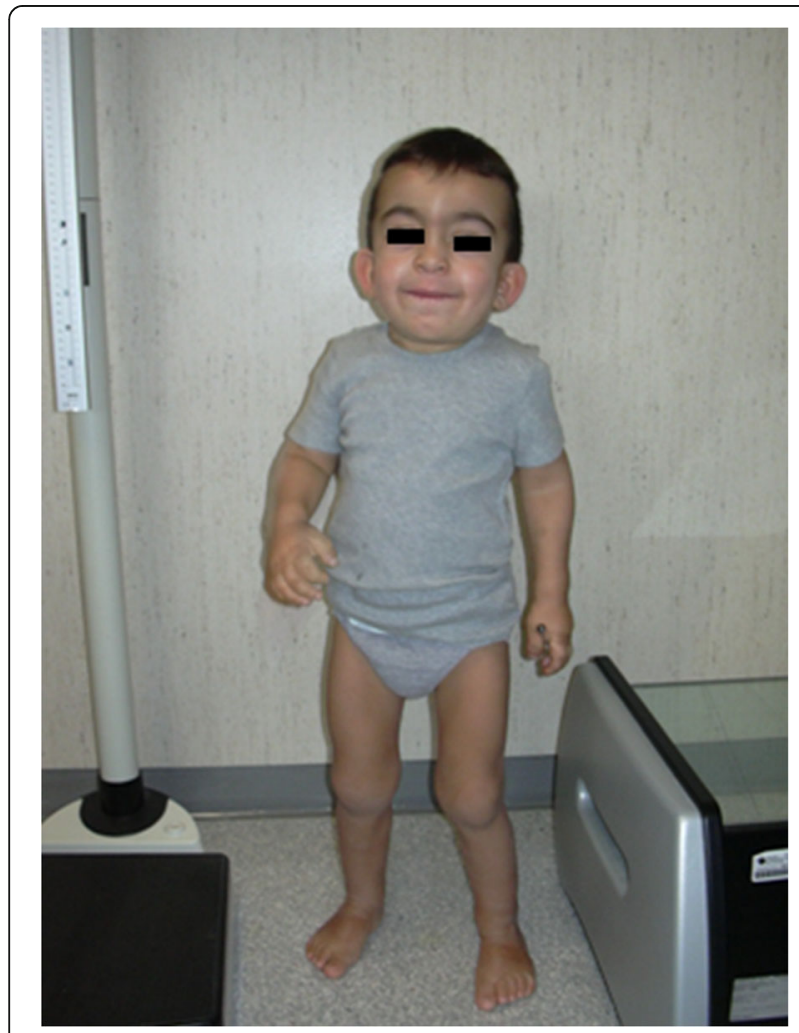

Fig. 9 MPS VI. A 2-year-old boy with evident coarse face and skeletal dysostosis and progressive skeletal damage, named dysostosis multiplex, becomes evident. This skeletal dysplasia includes hip dysplasia with dysplastic femoral head, abnormal vertebral bodies, irregular clavicles, hypoplastic distal ulna and radius, and dysplastic and short metacarpal bones; carpal and tarsal bones are hypoplastic and have an irregular profile. Growth velocity often slows after the first year of life, with a final height generally lower than $120 \mathrm{~cm}$. As in other MPS, an early sign is coarse facial features. In addition, patients have a typical protruding abdomen, hepatomegaly, umbilical and/or inguinal hernia, and heart involvement in early infancy. Although no primary intellectual disability is present, hydrocephalus can be seen in some patients with consequent intracranial hypertension and papilledema. Pectus carinatum, stiff and contracted joints, scoliosis and kyphosis, and cervical spinal cord compression worsen with time [32].

In summary, MPS IV and VI do not show CNS involvement. MPS IV is a unique MPS in showing joint laxity, while severe MPS VI onset is similar to that observed in MPS I.

\section{Conclusions}

Symptoms and signs suggestive of MPS can lead to misdiagnosis or be underestimated if not considered as a 
continuum. Although many of these features have other possible causes, they should promptly arouse suspicion of MPS in any infant or child presenting with one or more of them. Referring these patients to a metabolic centre will allow the recognition of these disorders at a very early stage.

We want to stress the need for increased awareness of MPS among paediatricians and other specialists to shorten the still existing diagnostic delay [33]. While many attempts have been made to set affordable newborn screenings for this group of disorders, a timely diagnosis is mandatory in view of a possible early treatment when available. Many pilot studies on newborn screening for MPS I are at present ongoing with the aim of detecting the disease in a pre-symptomatic phase. This will bring new pros and cons which should be carefully evaluated over the upcoming years [34] (for a deeper discussion of newborn screening in MPS, see Donati et al. in this Supplement [35]).

\section{Abbreviations}

ADHD: Attention deficit hyperactivity disorder; ASB: Arylsulfatase B; CNS: Central nervous system; CS: Chondroitin sulphate; DS: Dermatan sulphate; GAG: Glycosaminoglycan; HS: Heparan sulphate; KS: Keratan sulphate; MPS: Mucopolysaccharidosi(e)s; NIHF: Non-immune hydrops fetalis

\section{Acknowledgements}

The authors wish to thank the Italian MPS family Association ONLUS (AIMPS) and each patient and family who were followed at their centers. CG wishes to thank Fondazione Pierfranco and Luisa Mariani, Milano, for providing financial support for clinical assistance to metabolic patients and Mrs Vera Marchetti for her smart work as a secretary of the Metabolic Unit in Monza.

\section{Funding}

The publication costs for this paper in the IJP supplement were made possible with unconditional financial support from BioMarin, Sanofi Genzyme, and Shire. The sponsors had no input into the content of articles, which were independently prepared by the authors and have undergone the journal's standard peer-review process.

\section{About this supplement}

This article has been published as part of Italian Journal of Pediatrics, Volume 44 Supplement 2, 2018: Mucopolysaccharidoses: state of the art. The full contents of the supplement are available online at https:/ijponline.biomedcentral.com/ articles/supplements/volume-44-supplement-2.

\section{Authors' contributions}

Each author wrote a part of the first draft. AF and MDR revised and completed the text. All authors read and approved the final paper.

\section{Consent for publication}

The patients or their parents/guardians gave written consent for publication of their pictures.

\section{Competing interests}

The authors declare that they have no competing interests.

\section{Publisher's Note}

Springer Nature remains neutral with regard to jurisdictional claims in published maps and institutional affiliations.

\section{Author details}

'Department of Pediatrics, Fondazione MBBM, San Gerardo Hospital, Monza, Italy. ${ }^{2}$ Unit of Rare Diseases, Department of Pediatrics, Gaslini Institute, Genoa, Italy. ${ }^{3}$ Regional Referral Centre for Metabolic Diseases, Pediatric Clinic,
Department of Clinical and Experimental Medicine, University of Catania, AOU Policlinico VE, Via Santa Sofia 78, 95123 Catania, Italy.

Published: 16 November 2018

\section{References}

1. Rigoldi M, Verrecchia E, Manna R, Mascia MT. Clinical hints to diagnosis attenuated forms of mucopolysaccharidosis. Ital J Pediatr. 2018. https://doi.org/10.1186/s13052-018-0551-4.

2. Kiely BT, Kohler JL, Coletti HY, Poe MD, Escolar ML. Early disease progression of hurler syndrome. Orphanet J Rare Dis. 2017;2:32.

3. Wraith JE, Jones S. Mucopolysaccharidosis type I. Pediatr Endrocrinol Rev. 2014;12(Suppl 1):102-6.

4. Parini R, Jones SA, Harmatz PR, Giugliani R, Mendelsohn NJ. The natural history of growth in patients with Hunter syndrome: data from the Hunter Outcome Survey (HOS). Mol Genet Metab. 2016;117(4):438-46.

5. Bertola F, Filocamo M, Casati G, Mort M, Rosano C, Tylki-Szymanska A, et al. IDUA mutational profiling of a cohort of 102 European patients with mucopolysaccharidosis type I: identification and characterization of 35 novel a-Liduronidase (IDUA) alleles. Hum Mutat. 2011:32(6):E2189-210.

6. Khan SA, Peracha H, Ballhausen D, Wiesbauer A, Rohrbach M, Gautschi M, et al. Epidemiology of mucopolysaccharidoses. Mol Genet Metab. 2017;121:227.

7. Neufeld EF, Muenzer J. The mucopolysaccharidoses. In: Valle D, Beaudet AL, Vogelstein B, Kinzler KW, Antonarakis SE, Ballabio A, et al., editors. The online metabolic and molecular bases of inherited disease (OMMBID). Chapter. New York, NY: McGraw-Hill; 2015. p. 136.

8. Scarpa M, Almassy Z, Beck M, Bodamer O, Bruce I, De Meirleir L, et al. Mucopolysaccharidosis type II: European recommendations for the diagnosis and multidisciplinary management of a rare disease. Orphanet $\rfloor$ Rare Dis. 2011:6:72.

9. Lonardo F, Di Natale P, Lualdi S, Acquaviva F, Cuoco C, Scarano F, et al. Mucopolysaccharidosis type II in a female patient with a reciprocal X;9 translocation and skewed X chromosome inactivation. Am J Med Genet A. 2627;164A:2014

10. Martin R, Beck M, Eng C, Giugliani R, Harmatz P, Muñoz V, et al. Recognition and diagnosis of mucopolysaccharidosis II (hunter syndrome). Pediatrics. 2008;121:e377-86

11. Sheth J, Mistri M, Shah K, Chaudhary M, Godbole K, Sheth F. Lysosomal storage disorders in nonimmune hydrops fetalis (NIHF): an Indian experience. JIMD Rep. 2016; 35:47-52.

12. Beck M, Arn P, Giugliani R, Muenzer J, Okuyama T, Taylor J, et al. The natural history of MPS I: global perspectives from the MPS I Registry. Genet Med. 2014:16(10):759-65.

13. Young ID, Harper PS. The natural history of the severe form of Hunter's syndrome: a study based on 52 cases. Dev Med Child Neurol. 1983; 25(4):481-9.

14. Clarke LA. Mucopolysaccharidosis type I. In: Pagon RA, Adam MP, Ardinger $H H$, Wallace SE, Amemiya A, Bean $L H$, et al., eds. GeneReviews [internet]. Seattle (WA): University of Washington, Seattle; 1993-2017. 31 Oct 2002 (updated 201611 Feb 2016).

15. Mendelsohn NJ, Harmatz P, Bodamer O, Burton BK, Giugliani R, Jones SA, et al. Hunter outcome survey investigators. Importance of surgical history in diagnosing mucopolysaccharidosis type II (Hunter syndrome): data from the Hunter Outcome Survey. Genet Med. 2010;12(12):816-22.

16. Scarpa M. Mucopolysaccharidosis type II. In: Pagon RA, Adam MP, Ardinger $\mathrm{HH}$, Wallace $\mathrm{SE}$, Amemiya A, Bean $\mathrm{LH}$, et al., eds. GeneReviews [internet]. Seattle (WA): University of Washington, Seattle; 1993-2017. 6 Nov 2007 (updated 26 Mar 2015).

17. Dam TA. Microcephaly in hurler syndrome. Clin Pediatr (Phila). 1997; 36(1):51-2.

18. Tylki-Szymańska A. Mucopolysaccharidosis type II, Hunter's syndrome. Pediatr Endocrinol Rev. 2014:12(Suppl 1):107-13.

19. Sly WS, Quinton BA, McAlister WH, Rimoin DL. Beta glucuronidase deficiency: report of clinical, radiologic, and biochemical features of a new mucopolysaccharidosis. J Pediatr. 1973;82:249-57.

20. Pfeiffer RA, Kresse H, Bäumer N, Sattinger E. Beta-glucuronidase deficiency in a girl with unusual clinical features. Eur J Pediatr. 1977;126:155-61.

21. Montaño AM, Lock-Hock N, Steiner RD, Graham BH, Szlago M, Greenstein R, et al. Clinical course of Sly syndrome (mucopolysaccharidosis type VII). J Med Genet. 2016;53(6):403-18. 
22. Nidiffer FD, Kelly TE. Developmental and degenerative patterns associated with cognitive, behavioural and motor difficulties in the Sanfilippo syndrome: an epidemiological study. J Ment Defic Res. 1983;27:185-203.

23. Wijburg FA, Wegrzzyn G, Burton BK, Tylki-Szymańska A. Mucopolysaccharidosis type III (Sanfilippo syndrome) and misdiagnosis of idiopathic developmental delay, attention deficit hyperactivity or autism spectrum disorder. Acta Paediatr. 2013;102:462-70

24. Bax MC, Colville GA. Behaviour in mucopolysaccharide disorders. Arch Dis Child. 1995;73(1):77-81.

25. Valstar MJ, Bruggenwirth HT, Olmer R, Wevers RA, Verheijen FW, Poorthuis BJ, et al. Mucopolysaccharidosis type IIIB may predominantly present with an attenuated clinical phenotype. J Inherit Metab Dis. 2010;33:759.

26. Heron B, Mikaeloff $Y$, Froissart R. Incidence and natural history of mucopolysaccharidosis type III in France and comparison with United Kingdom and Greece. Am J Med Genet 2010;research article:58-68.

27. Hendriksz CJ, Harmatz P, Beck M, Jones S, Wood T, Lachman R, et al. Review of clinical presentation and diagnosis of mucopolysaccharidosis IVA. Mol Genet Metab. 2013;110:54-64.

28. Caciotti A, Garman SC, Rivera-Colon Y, Procopio E, Catarzi S, et al. GM1 gangliosidosis and Morquio $\mathrm{B}$ disease: an update on genetic alterations and clinical findings. Biochim Biophys Acta. 2011;1812:782.

29. Montano AM, Tomatsu S, Gottesman GS, Smith M, Orii T. International Morquio a registry: clinical manifestation and natural course of Morquio a disease. J Inherit Metab Dis. 2007:30:165-74.

30. Hendriksz CJ, Berger KI, Giugliani R, Harmatz P, Kampmann C, Mackenzie $W G$, et al. International guidelines for the management and treatment of Morquio A syndrome. Am J Med Genet A. 2015;167A(1):11-25.

31. Trowbridge JM, Gallo RL. Dermatan sulfate: new functions from an old glycosaminoglycan. Glycobiology. 2002;12:117R-25R.

32. Valayannopoulos V, Nicely H, Harmatz P, Turbeville S. Mucopolysaccharidosis VI. Orphanet J Rare Dis. 2010;5:5.

33. Fiumara A, Sorge G, Del Campo G, Trigilia C, Barone R. Hurler disease (MPS): evidence for the need of increased awareness by different specialists. Mol Genet Metab. 2013;108:540.

34. Peake RW, Bodamer OA. Newborn screening for lysosomal storage disorders. J Pediatr Genet. 2017;6(1):51-60.

35. Donati M, Pasquini E, Spada M, Polo G, Burlina A. Newborn screening in mucopolysaccharidoses. Ital J Pediatr. 2018. https://doi.org/10.1186/s13052018-0552-3.

\section{Ready to submit your research? Choose BMC and benefit from:}

- fast, convenient online submission

- thorough peer review by experienced researchers in your field

- rapid publication on acceptance

- support for research data, including large and complex data types

- gold Open Access which fosters wider collaboration and increased citations

- maximum visibility for your research: over $100 \mathrm{M}$ website views per year

At $\mathrm{BMC}$, research is always in progress.

Learn more biomedcentral.com/submissions 